\title{
Decrease of Perforin Expressing Lymphocytes after On-Pump Coronary Artery Bypass Grafting Surgery Irrespective of Carbohydrate Preoperative Oral Feeding
}

\author{
Jadranko Sokolic, MD, PhD,${ }^{1}$ Danijel Knezevic, MD,${ }^{1}$ Janja Kuharic, MD, PhD, ${ }^{1}$ Igor Medved, MD, PhD, ${ }^{2}$ \\ Alan Sustic, MD, PhD, ${ }^{1}$ Zeljko Zupan, MD, PhD,${ }^{1}$ Gordana Laskarin, $\mathrm{MD}, \mathrm{PhD},{ }^{3,4}$ Tomislav Tadin, $\mathrm{MD}, \mathrm{PhD},{ }^{5}$ \\ Vlatka Sotosek Tokmadzic, $\mathrm{MD}, \mathrm{PhD}^{1}$ \\ Departments of ${ }^{1}$ Anesthesia, Resuscitation, Emergency and Intensive Care Medicine, ${ }^{2}$ Surgery, and ${ }^{4}$ Physiology and Immunology, \\ Faculty of Medicine, University of Rijeka; ${ }^{3}$ Division of Cardiology, Hospital for Medical Rehabilitation of the Hearth and Lung Diseases \\ and Rheumatism "Thalassotherapia-Opatija," Opatija; ${ }^{5}$ Ultrasound Diagnostic Service, Health Centre Rijeka, Rijeka, Croatia
}

\section{ABSTRACT}

Background: Coronary artery bypass grafting (CABG) surgery continues to be the gold standard for treating the patients with coronary artery disease. CABG surgery can be performed on or off cardiopulmonary bypass, termed as onpump or off-pump CABG, respectively. It has been shown that CABG surgery, preferably on-pump CABG surgery, leads to the changes of cell immunity during perioperative and early postoperative period. The mechanisms of regulation of the immune response in patients during and early after surgical revascularization are not fully understood.

The aim of this study was to investigate the influence of carbohydrate preoperative oral feeding on frequency and perforin expression in peripheral blood lymphocytes in patients after on- or off-pump CABG surgery in early postoperative period.

Patients and methods: In this prospective clinical study, 80 patients scheduled for CABG surgery were included in the study. The patients were randomly allocated into four groups (20 in each group): patients in Group 1 underwent on-pump CABG and did not receive carbohydrate preoperative oral feeding; patients in Group 2 underwent on-pump CABG and were preoperatively fed; patients in Group 3 underwent offpump CABG and did not receive carbohydrate preoperative oral feeding; while patients in Group 4 underwent off-pump CABG and received carbohydrate preoperative oral feeding. Blood samples were collected immediately before (T1), 24 (T2) and 72 (T3) hours after the surgery. Peripheral blood mononuclear cells were isolated by gradient centrifugation and simultaneously labelled by antigens using fluorochromeconjugated monoclonal antibodies. Frequency of T lymphocytes, NK and NKT cells, their subsets as well as their perforin expression were detected, and analyzed by flow cytometry.

Results: There was significant decrease in frequency of $\mathrm{CD}^{+}$and $\mathrm{CD}^{+}{ }^{+} \mathrm{CD}^{+}{ }^{+}$cells, as well as perforin expressing

Received March 17, 2018; accepted March 11, 2019.

Correspondence: Vlatka Sotosek Tokmadzic, MD, PhD, Anesthesia, Resuscitation, Emergency and Intensive Care Medicine, Faculty of Medicine, University of Rijeka, 51000 Rijeka, B. Branchetta 20, Croatia; (e-mail: vlatkast@medri.uniri.br).
$\mathrm{CD}^{+}{ }^{+} \mathrm{CD} 8{ }^{+}$cells in patients who underwent on-pump $\mathrm{CABG}$ in comparison to patients who underwent off-pump CABG 24 hours after the surgery. Carbohydrate preoperative oral feeding did not effect changes in lymphocytes subpopulations and perforin expression at any time point.

Conclusion: Decreases of $\mathrm{CD}^{+}{ }^{+}$cells on account of $\mathrm{CD}^{+}{ }^{+} \mathrm{CD} 4{ }^{+}$subsets, and perforin expressing cells on account of $\mathrm{CD}^{+}{ }^{+} \mathrm{CD} 8{ }^{+}$perforin ${ }^{+}$cells were found in patients who had undergone on-pump CABG, but not in patients who had undergone off-pump CABG surgery, irrespectively of carbohydrate preoperative oral feeding.

\section{INTRODUCTION}

Coronary artery disease is the most common heart disease, a major cause of hospital admission and the main single cause of death worldwide, which represents a serious public health issue [Solomon 2013; Finegold 2012]. It often occurs as a result of atherosclerotic plaques along the inner wall of the coronary arteries, leading to a decrease in blood flow and reducing the delivery of oxygen and nutrients to the heart muscle. The underlying process of ischemic heart disease is endothelial dysfunction and chronic progressing, a pro-inflammatory, and potentially lethal event, which positively correlates with the degree of ischemia and apoptosis of cardiomyocytes and culminates with myocardial necrosis in acute myocardial infarction (AMI) onset [Akasaka 2006]. A significant increase of the pro-inflammatory cytokines such as interleukin (IL)-1 beta, IL-6, IL-8, IL-18, and high sensitive C-reactive protein (hsCRP) were found in patients with coronary artery disease [Bujak 2009; Martins 2006]. Activated endothelium is the main source of pro-inflammatory cytokines and chemokines, which attract leukocyte subsets (neutrophils, lymphocytes, monocytes, NK cells) from the blood stream, activate them, and encourage their entry into the vascular wall (atheroma) of myocardium at the site of acute ischemic damage, respectively [Bujak 2009; Allavena 1997]. Activated T cells were found in peripheral blood and plaques of patients with coronary artery disease [Zal 2008] as well as myocardium after AMI [Abbate 2008]. It is speculated that IL-15 recruits T lymphocytes and NK cells in infarction affected myocardium [Persic 2012], as 
it has been shown for atherosclerotic plaque [Allavena 1997]. IL-15 strongly increases cytotoxic activity of T lymphocytes and $\mathrm{CD} 56^{\text {+dim }}$ and $\mathrm{CD} 56^{+ \text {tright }}$ subpopulation of $\mathrm{NK}$ cells and upregulates the expression of cytotoxic agents, such as perforin [Perera 2012; Tokmadzic 2002] in the cytoplasmic granules [Chávez-Galán 2009; Pipkin 2007]. In synergy with IL-2, IL-15 enhances the production of pro-inflammatory nterferon gamma (IFN $\gamma$ ), tumor necrosis factor alpha (TNF $\alpha$ ) in cytotoxic NK cells [Perera 2012]. By the activation of cytotoxic T and NK cells, perforin is ejected from the cytotoxic granules by exocytosis, installed, and polymerized in the membrane of target cells in the presence of calcium ions in order to create cylindrical pores [Chávez-Galán 2009; Pipkin 2007]. Through the pores, water and proapoptotic molecules enter into the target cell causing its death by necrosis or apoptosis, respectively [Chávez-Galán 2009; Pipkin 2007]. Surgical revascularization, which bridges the narrowing or blockage of a coronary artery, remains the gold standard for the treatment of patients with significant stenosis of the main trunk of the left coronary artery, for the patients with multivessel coronary artery disease [Takayama 2010; Buxton 2013] and for the patients with AMI that cannot be controlled by medication or primary percutaneous coronary intervention [Jeremias 2009]. Surgical coronary revascularization, performed with on-pump coronary artery bypass grafting (CABG) surgery, supports perioperative and early postoperative systemic inflammatory immune response in these patients more than off-pump CABG surgery [Orhan 2007]. At the peak of the local inflammatory events in patients with coronary artery disease, the possibility for the development of AIM in early postoperative period increases dramatically [Orhan 2007]. Additionally, strong systemic inflammatory response (SIRS) causes an acute confusional state due to hyperresponsivity of the neuro-immune cells to cytokines stimulation in the significant number of postoperative cardiac surgery patients [Kazmierski 2014]. These conditions significantly affect treatment outcome. However, mechanisms of regulation of the immune response in patients during and early after surgical revascularization are still not fully understood.

There is an increasing body of evidence that show the importance of optimal preoperative and postoperative nutrition on the immune status of patients [Anastasilakis 2013; Molfino 2014]. It is a common practice to stop oral ingestion of drinks and food $6 \mathrm{~h}$ before surgery [Ludwig 2013]. However, the negative impact of preoperative fasting on postoperative recovery of patients has already been recognized [Anastasilakis 2013].

To evaluate whether carbohydrate preoperative oral feeding affects cell immunity, the aim of this study was to analyze and compare the influence of preoperative oral feeding on frequency in phenotype and perforin expression of $\mathrm{T}$ lymphocytes, NK, and NKT cells in peripheral blood of patients who underwent on-pump and off-pump CABG surgery.

\section{PATIENTS AND METHODS}

The study protocol was approved by the local ethics committee of the Clinical Hospital Centre Rijeka Rijeka, Croatia

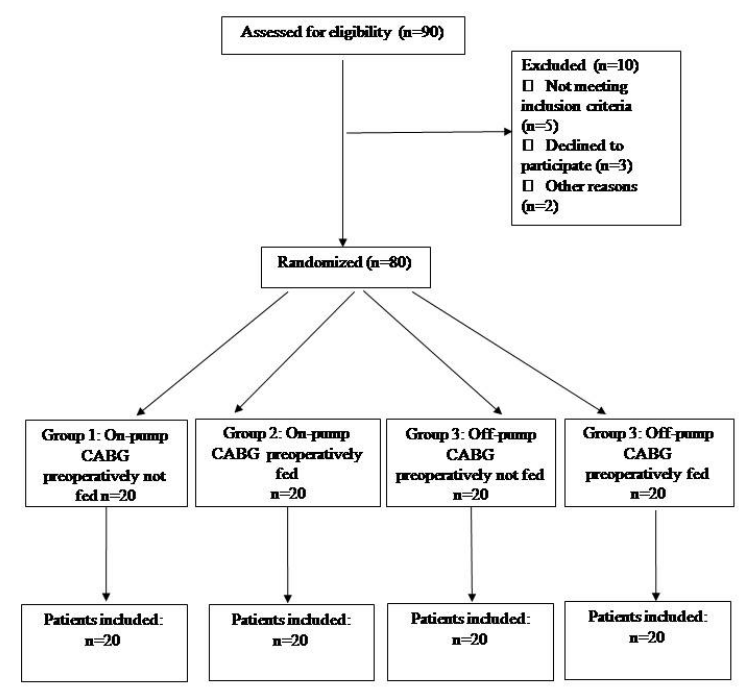

Figure 1. Flowchart of the study.

and the Faculty of Medicine, University of Rijeka (Rijeka, Croatia) and was carried out according to the World Medical Association criteria in the declaration of Helsinki. To all the patients included in the study, the study's protocol was explained and signed informed consent was given.

Eighty patients, well-nourished according to Subjective Global Assessment of Nutritional Status (SGA)-A and scheduled for CABG surgery due to ischemic heart disease were admitted to the Clinical Hospital Centre Rijeka, Croatia, and were included in this prospective randomized study. Forty patients underwent on-pump CABG surgery and 40 patients underwent off-pump CABG surgery. Indications for CABG were consistent with the European Society of Cardiology (ESC) and the European Association for Cardio-Thoracic Surgery (EACTS) [Windecker 2014]. Patients in each group were randomly allocated by the Datlnf Ranolist computer program (Datlnf, Tubingen, Germany) to one of the groups according to carbohydrate preoperative oral feeding: Group 1 underwent on-pump CABG and did not receive carbohydrate preoperative oral feeding; patients in Group 2 underwent on-pump CABG and were preoperatively fed; patients in Group 3 underwent off-pump CABG and did not receive carbohydrate preoperative oral feeding; while patients in Group 4 underwent off-pump CABG and received carbohydrate preoperative oral feeding (Figure 1). Patients allocated to Group 2 and Group 4 were given $200 \mathrm{~mL}$ of enteral nutrition formula "PreOp" (Nutricia, Dublin, Ireland) two hours before the surgery, while patients in Group 1 and Group 3 did not receive preoperative oral feeding. "PreOp" is a drink high in carbohydrates, and contains $0 \mathrm{~g}$ of proteins, $12.6 \mathrm{~g}$ of sugars and $0 \mathrm{~g}$ of fats in $100 \mathrm{~mL}$.

Exclusion criteria from the study: patients younger than 18, older than 75, SGA-B (malnourished) SGA-C (severely malnourished), patients who received blood transfusion, patients with immunological and malignant disease, diabetes 
Demographic and Clinical Parameters of Patients*

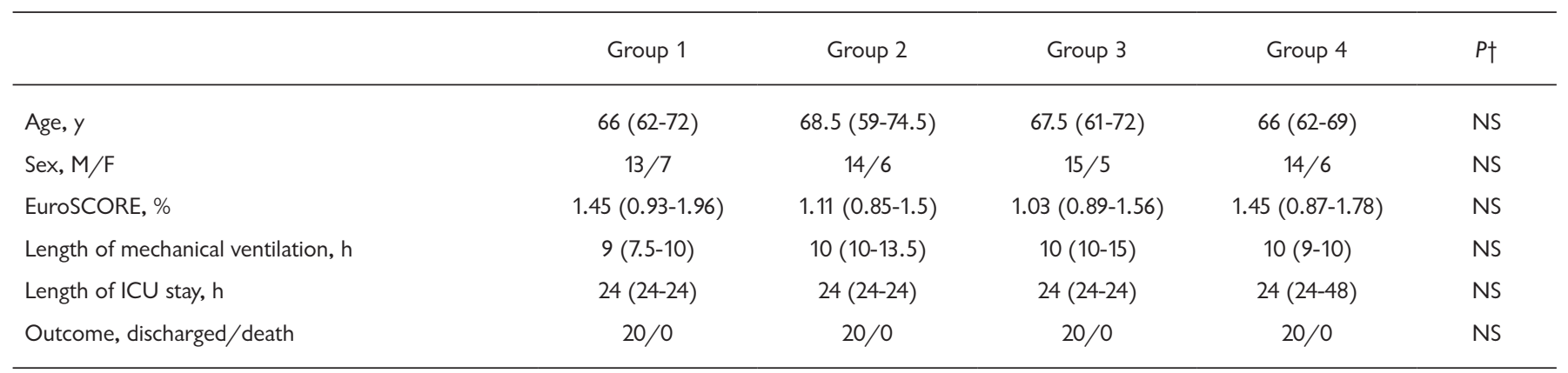

*Group 1: patients who underwent on-pump CABG and did not reveive perioperative nutrition support. Group 2: patients who underwent on-pump CABG and were preoperatively fed. Group 3: patients who underwent off-pump CABG and did not reveive perioperative nutrition support. Group 4: patients who underwent off-pump CABG and received preoperative nutrition support. Data are presented as median (25th-75th percentile).

$\dagger$ NS: $P$ values between groups were not statistically significant.

mellitus, renal or heart failure, and those with a CABG time over 110 minutes.

\section{Anesthesia and Surgical Procedures}

General balanced anesthesia with sedative midazolam (Dormicum, Roche, Basel, Switzerland), opioid sufentanyl (Renaudin, Itxassou, France) and muscle relaxant rocuronium bromide (Esmeron, Merck Sharp \& Dohme, Kenilworth, New Jersey, USA) was administrated in all the patients. All patients were endotracheally intubated and mechanically ventilated.

Surgical procedure was carried out through median sternotomy. On-pump CABG surgery was performed using Terumo System 1 (Terumo, Tokyo, Japan) with affinity fusion oxygenator filter (Medtronic, Minneapolis, MN, USA). Mean arterial pressure was maintained between 50 and $80 \mathrm{mmHg}$ and blood flow was kept at $2.4 \mathrm{~L} / \mathrm{min}$ per $\mathrm{m}^{2}$. Moderate systemic hypothermia of $32-34^{\circ} \mathrm{C}$ was applied. Calafiore blood cardioplegia was applied for cardiac preservation [Calafiore 2003]. Heparin (300 IU/kg) was administrated before the onset of CABG and was monitored by means of the activated clotting time $(>400$ s). Protamine chloride was administrated when all anastomoses were completed to reverse to a normal activated clotting time. Off-pump CABG surgeries were performed on the beating heart using the Octopus IV system (Medtronic, Minneapolis, $\mathrm{MN}, \mathrm{USA}$ ) and the heparinization was obtained by $150 \mathrm{IU} / \mathrm{kg}$ of heparine. After the surgery all patients were admitted to the intensive care unit for postoperative care.

\section{Blood Sampling and Isolation of Peripheral Blood Monocytes}

Twenty milliliters of venous peripheral blood samples were obtained from each patient before (T1), 24 (T2) and 72 (T3) hours after surgery. Blood samples were overlaid onto density gradient media (Lymphoprep, Fresenius Kabi, Oslo, Norway) and mononuclear cells were isolated from peripheral blood by centrifugation at $600 \times \mathrm{g}$ for $20 \mathrm{~min}$. Viability of the isolated cells was assessed with propidium iodide $0.5 \mu \mathrm{g} / \mathrm{mL} / 10^{6}$ cells (Sigma-Aldrich, Taufkirchen Germany) and a flow cytometer
(FACSCalibur, Becton Dickinson, San Jose, CA, USA) analysis, and was over $98 \%$.

\section{Cell Surface and Intracellular Staining of Peripheral Blood Lymphocytes}

Isolated mononuclear cells were incubated with $10 \%$ heat-inactivated human $\mathrm{AB}$ serum at $21^{\circ} \mathrm{C}$ for $20 \mathrm{~min}$ to block non-specific Fc receptor binding. Then the cells were washed in fluorescence-activated cell sorter (FACS) buffer [NaCl $(140 \mathrm{mM}), \mathrm{KH}_{2} \mathrm{PO}_{4}(1.9 \mathrm{mM}), \mathrm{Na}_{2} \mathrm{HPO}_{4}(16.5 \mathrm{mM})$, $\mathrm{KCl}(3.75 \mathrm{mM})$ (all from Kemika, Zagreb, Croatia), $\mathrm{Na}_{2}$ EDTA (0.96 mM) (Fluka, Buchs, Switzerland), $\mathrm{NaN}_{3}(1.5$ $\mathrm{mM}$ ) (Difco, Detroit, Michigan)] and were fixed with $4 \%$ paraformaldehyde $\mathrm{pH} 7.4$ for $10 \mathrm{~min}$ at $21^{\circ} \mathrm{C}$. Samples for intracellular staining were washed in FACS buffer and permeabilized with saponin buffer $(0.1 \%$ saponin, Sigma, Poole, Dorset, USA), $2 \%$ goat serum in phosphate buffered saline (PBS) $\left[\mathrm{NaCl} 8 \mathrm{~g}, \mathrm{KCl} 0.2 \mathrm{~g}, \mathrm{Na}_{2} \mathrm{HPO}_{4} \times 12 \mathrm{H}_{2} 02.87 \mathrm{~g}\right.$ and $\mathrm{KH}_{2} \mathrm{PO}_{4} 0.2 \mathrm{~g}$ (all from Kemika, Zagreb, Croatia)) dissolved in $1 \mathrm{~L}$ of distilled water)] for $20 \mathrm{~min}$ at $21^{\circ} \mathrm{C}$. After 20 minutes of incubation mouse $\delta \mathrm{G} 9 \mathrm{IgG} 2 \mathrm{~b}$ anti-perforin monoclonal antibody or isotype matched control (mouse MA-21, IgG2b, Biosciences Erembodegen, Belgium) directly conjugated with fluorescein isothiocyanate (FITC) was added ( $2 \mu \mathrm{L}$ each) to the sample and incubated for $30 \mathrm{~min}$ at $+4^{\circ} \mathrm{C}$. The abundance of antibodies was washed by saponin buffer. To restore the membrane integrity, samples were resuspended in $1 \mathrm{~mL}$ FACS buffer. For surface labelling, samples were incubated with the combination of CyCrome phycoerithryn-5 (Cy-PE5) conjugated anti-CD3 (mouse UCHT1, IgG1) and phycoerithryn (PE) conjugated anti-CD4 mAb (mouse RPA-T4, IgG1), Cy-Pe5 anti-CD3 and PE anti-CD8 (mouse RPA-T8, IgG1) or $\mathrm{Cy}-\mathrm{Pe} 5$ anti-CD3 and PE anti-CD56 (mouse B159, IgG1). Directly conjugated FITC, PE or CY-PE5 mouse isotype match antibodies (Bioscience, Erembodegen, San Diego, CA, USA) were used as the controls. After incubation all samples were fixed in $400 \mu \mathrm{L}$ of $2 \%$ paraformaldehyde and acquired by flow cytometer (FACSCalibur, Beckton-Dickinson, Heidelberg, Germany). 

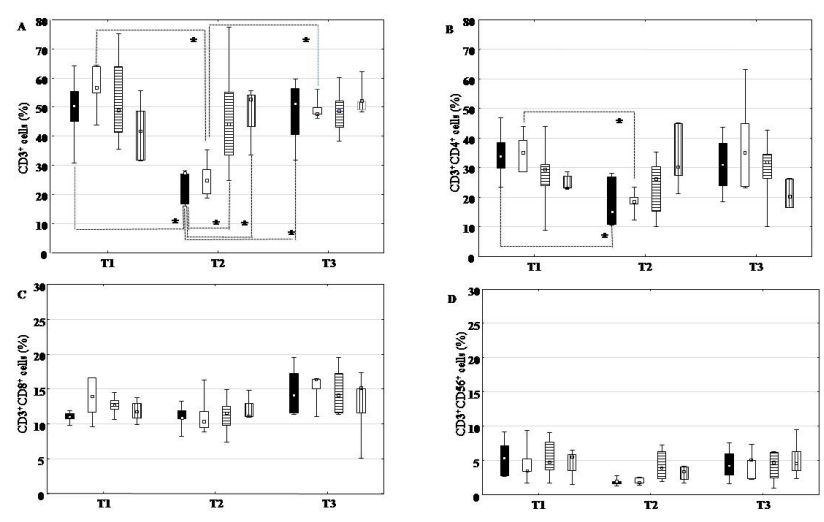

Figure 2. Frequencies of $\mathrm{CD}^{+} \mathrm{CD} 56^{-}(\mathrm{A}), \mathrm{CD}^{+} \mathrm{CD} 4^{+} \mathrm{CD} 56^{-}(\mathrm{B})$, $\mathrm{CD}^{+}{ }^{+} \mathrm{CD} 8^{+} \mathrm{CD} 56^{-}(\mathrm{C})$, and $\mathrm{CD} 3^{+} \mathrm{CD} 56^{+}(\mathrm{D})$ cells in peripheral blood

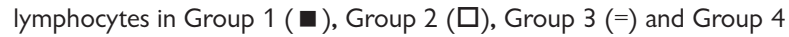
(I) at times points T1 (before surgery), T2 (24 h after surgery), T3 (72 $h$ after surgery). Data are presented as median (), 25th-75th percentile $(\square)$, non-outlier range $(\mathrm{I})$, and outliers $(\circ)$. Statistical significance: $* P<.05$.

\section{Statistical Analysis}

Statistical analysis was carried out by data analysis software platform Statistica 13.3 (TIBCO Software, Palo Alto, CA, USA). Continuous variables were presented as $25-75 \%$ (25th-75th percentile) values and categorical variables were presented as numbers. Kolmogorov-Smirnov test was used to test the normality of continuous variables. Since the data had not shown normal distribution, the Kruskal-Wallis nonparametric test was used to analyze the differences among groups and Mann-Whitney $\mathrm{U}$ tests were used to compare the data between groups post-hoc. Friedman's test and the post-hoc Wilcoxon rank sum test were used to compare time points within groups. When multiple comparisons were made, Bonferroni adjustments were applied. $\mathrm{G}^{*}$ power program (G*Power 3.1.9.2 Software 2014, University of Dusseldorf, Germany) was applied to estimate the sample size. The predictive sample size was calculated for nonparametric tests. The total sample size was 15 respondents and 8 respondents per group with a two-sided confidence interval of 0.95 and a desired power of 0.80 . A difference was judged to be statistically significant at a $P$ value $<.05$.

\section{RESULTS}

Demographic and clinical data are shown in Table 1. Patients did not differ according to age, sex, EuroSCORE, length of mechanical ventilation, length of intensive care unit (ICU) stay, and outcome.

\section{Dynamic Changes in Frequency of Lymphocyte Subsets in Peripheral Blood of the Patients}

The frequency of $\mathrm{CD}^{+}$lymphocytes did not mutually differ before surgery (time point T1) among groups (Figure 2, A). Significant decrease in frequency of $\mathrm{CD} 3^{+}$lymphocytes was

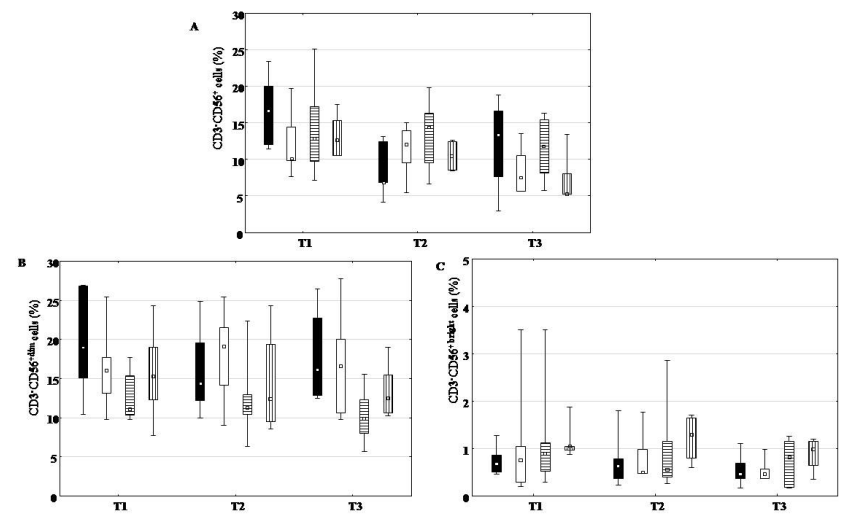

Figure 3. The frequency of all $C D 3{ }^{-} C D 56^{+}$NK cells $(A)$, their $C D 3$ CD56 $6^{\text {+dim }}(B)$, and $C D 3-C D 56^{\text {tbright }}(C)$ subsets within peripheral blood lymphocytes in Group $1(\mathbf{\square})$, Group $2(\square)$, Group $3(=)$ and Group 4 (I) at times points T1 (before surgery), T2 (24 h after surgery), T3 (72 $\mathrm{h}$ after surgery). Data are presented as median (), 25th-75th percentile $(\square)$, non-outlier range (I), and outliers $(\circ)$. Statistical significance: $* P<.05$.

found in Group 1 and Group 2 at time point T2 (24 hours after surgery) when compared to time point $\mathrm{T} 1$ and restored at time point T3 (Figure 2, A). The decreased frequency of $\mathrm{CD} 3{ }^{+}$cells in Group 1 and Group 2 at time point T2 was significantly lower when compared to Group 3 and Group 4 (Figure 2, A).

Additionally, the frequency of $\mathrm{CD}^{+}{ }^{+} \mathrm{CD}^{+}{ }^{+}$lymphocytes reduced in Group 1 and Group 2 at time point T2 when compared to time point T1 (Figure 2, B), while the frequency of $\mathrm{CD}^{+}{ }^{+} \mathrm{CD} 8{ }^{+}$lymphocytes (Figure $2, \mathrm{C}$ ) and $\mathrm{CD} 3{ }^{+} \mathrm{CD} 56^{+} \mathrm{NKT}$ cells (Figure 2, D) did not significantly change among groups at any time points. NK cells of $\mathrm{CD} 3{ }^{-} \mathrm{CD} 56^{+}$phenotype (Figure 3, A) and their subsets CD56 $6^{\text {+dim }}$ (Figure 3, B) and CD 56 $6^{\text {bright }}$ (Figure 3, C) did not significantly change.

\section{Dynamic Changes of Perforin Expression in Peripheral Blood Lymphocytes}

Decrease of total perforin positive lymphocytes was observed in Group 1 and Group 2 at time point $\mathrm{T} 2$ when compared to time point T1 (Figure 4). The decrease of perforin expressing CD3+ cells (Figure 5, A) and particularly their $\mathrm{CD}_{3}{ }^{+} \mathrm{CD} 8^{+}$perforin ${ }^{+}$ subset (Figure 5, C) contributed to the decrease of total perforin positive lymphocytes. $\mathrm{CD}^{+}{ }^{+} \mathrm{CD} 4{ }^{+}$perforin ${ }^{+}$cells (Figure $5, \mathrm{~B}), \mathrm{CD}^{+}{ }^{+} \mathrm{CD} 56^{+}$perforin ${ }^{+}$NKT cells (Figure 5, D), CD3 $\mathrm{CD}^{+} 6^{+}$perforin ${ }^{+} \mathrm{NK}$ cells (Figure 6, A) and their subsets CD56 $6^{\text {+dim }}$ (Figure 6, B) and CD56 ${ }^{\text {+bright }}$ (Figure 6, C) did not significantly differ at any time point.

\section{DISCUSSION}

On-pump CABG surgery is accompanied by an increased possibility of creating complications such as increased stress, the occurrence of SIRS with frequent myocardial tissue damage, and adverse effects on cognitive function in the patients [Orhan 2007]. The SIRS, but not the local 


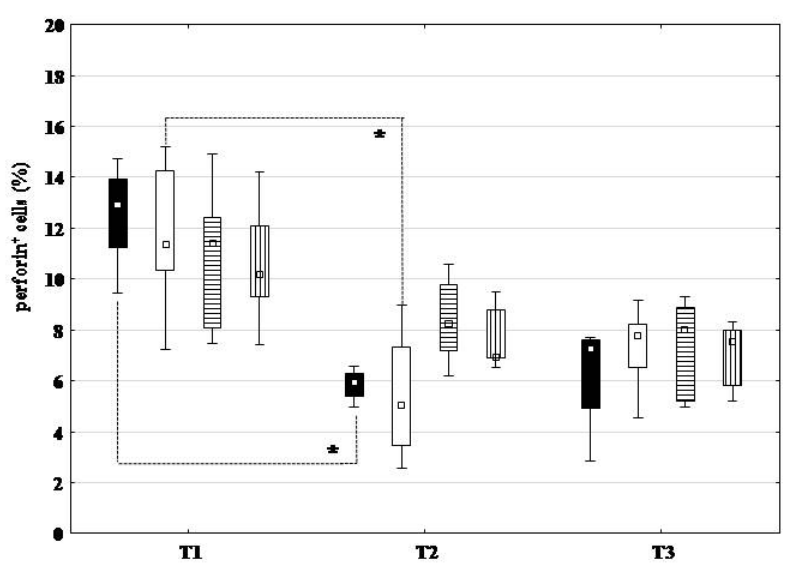

Figure 4. Frequencies of perforin-positive cells within peripheral blood lymphocytes in Group 1 ( $\mathbf{\square})$, Group 2 ( $\square$ ), Group 3 (=) and Group 4 (I) at times points T1 (before surgery), T2 (24 h after surgery), T3 (72 $\mathrm{h}$ after surgery). Data are presented as median ( $\square$ ), 25th-75th percentile $(\square)$, non-outlier range $(\mathrm{I})$, and outliers $\left({ }^{\circ}\right)$. Statistical significance: $* P<.05$.

inflammatory events in the myocardium, are reduced by using off-pump CABG surgical techniques [Takagi 2014]. This technique decreases stress and ensures faster postoperative recovery and shorter hospital stays [Takagi 2014], although it cannot be applied to all patients. Even with the improvement of surgical techniques, the perioperative and early postoperative myocardial infarction and acute confusional state remain the most common adverse complications that are associated with higher mortality and morbidity rates [Kazmierski 2014].

Surgical revascularization modulates immune parameters, primarily local and systemic cell-mediated immunity [Jankovicová 2008]. Lymphocytes activated by surgery are present in the circulation mediating primarily strong pro-inflammatory reaction [Jankovicová 2008]. We found significant decrease of $\mathrm{CD}^{+}$lymphocytes and their $\mathrm{CD}^{+}{ }^{+} \mathrm{CD} 4{ }^{+}$subsets 24 hours after surgery in patients who underwent on-pump but not off-pump CABG. It goes along with significant reduction in the number of $\mathrm{CD}^{+}$and $\mathrm{CD}^{+}$lymphocytes, CD19+ $\mathrm{B}$ lymphocytes, and $\mathrm{CD} 16^{+} \mathrm{CD} 56^{+} \mathrm{NK}$ cells in peripheral blood of the patients who underwent on-pump CABG immediately after surgery at day 1 [Akbas 2002]. However, it is not known whether the decrease of lymphocytes in peripheral blood is a consequence of their recruitment in the myocardium, although infiltration of activated $\mathrm{CD}^{+} \mathrm{T}$ cells was found in the myocardium at the site of ischemic myocardial necrosis and in remote areas of the heart with the evidence of apoptosis of patients with ischemic heart disease who died immediately, shortly (4 days), or later (4 months) after coronary thrombosis [Abbate 2008]. This indicates a local pro-inflammatory state and the role of $\mathrm{T}$ cells in apoptosis of cardiomyocytes with a consequent weakening of myocardial function and clinical exacerbation of the disease. Indeed, after the surgical revascularization, activated lymphocytes ( $\mathrm{T}$ and NK cells) could travel toward myocardial chemotactic factors, overexpressed during operation and ischemia/
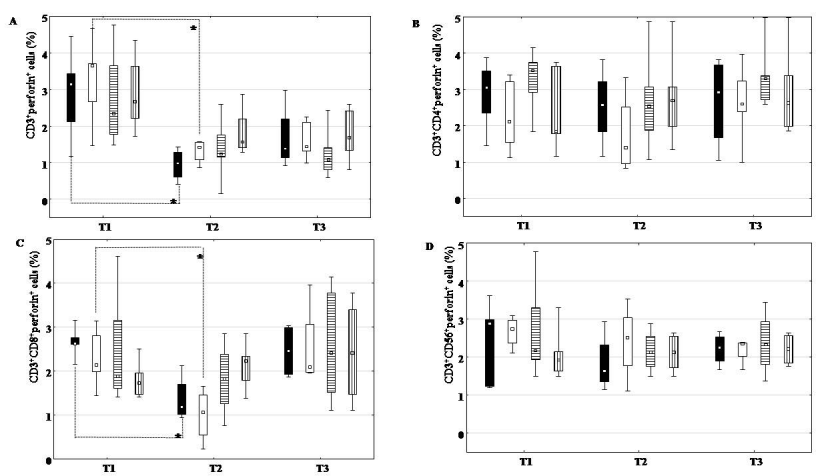

Figure 5. Frequencies of $\mathrm{CD}^{+}$perforin $^{+}(\mathrm{A}), \mathrm{CD}^{+} \mathrm{CD}^{+}$perforin ${ }^{+}(\mathrm{B})$, $\mathrm{CD}^{+}{ }^{+} \mathrm{CD} 8^{+}$perforin ${ }^{+}(\mathrm{C})$, and $\mathrm{CD}^{+} \mathrm{CD} 56^{+}$perforin ${ }^{+}$cells within peripheral blood lymphocytes in Group 1 ( $\mathbf{a})$, Group 2 (口), Group 3 (=) and Group 4 (I) at times points T1 (before surgery), T2 (24 h after surgery), T3 (72 h after surgery). Data are presented as median ( $\square$ ), 25 th-75th percentile $(\square)$, non-outlier range (I), and outliers $\left({ }^{\circ}\right)$. Statistical significance: $* P<.05$.

reperfusion injury [Zuidema 2010]. We have shown herein for the first time the decrease in the frequency of perforin positive circulating lymphocytes, predominantly of $\mathrm{CD}^{+} \mathrm{CD}^{+}$ phenotype, in patients who underwent on-pump CABG surgery 24 hours after the surgery. This suggests recruitment of perforin positive cells of cytotoxic phenotype in myocardial tissue attracted by locally produced factors such as cytokines [Laskarin 2012]. Indeed, more $\mathrm{CD}^{+}$and rare $\mathrm{CD}^{+} 6^{+}$perforin expressing lymphocytes were found in damaged myocardium after AMI [Persic 2012]. Recruited cells could be responsible for additional damage of myocardium while their lack in the circulation might increase susceptibility to infection. It could be one of the mechanisms that explain a higher incidence of AMI and infections in patients who underwent on-pump CABG surgery when compared to the patients who underwent off-pump surgical procedure [Møller 2014].

Recently, nutrition has been shown to influence immunity [Anastasilakis 2013]. Therefore, quantifying the nutritional status of patients by simple, fast, and reproducible methods, such as SGA scale, has become inevitable in contemporary clinical practice [Anastasilakis 2013]. Preoperative fasting is common in clinical practice in order to prevent aspiration of gastric contents in the perioperative period [Ludwig 2013], but it has negative impact on postoperative recovery of patients [Anastasilakis 2013]. Enteral nutrition allows physiological entering of nutrients into the body in a well-known quantity, reduces hepatobiliary complications and has favorable pharmacoeconomic parameters and advantages over parenteral [Anastasilakis 2013]. Even a slight amount of food in the intestinal lumen $(<200 \mathrm{~mL} / 24)$ can prevent atrophy of the intestinal villi and maintain the integrity of enterocytes [Anastasilakis 2013]. Therefore, new protocols for preoperative oral feeding are implemented in clinical practice, achieved by clear fluid rich in carbohydrates given to the patients once $2 \mathrm{~h}$ prior to surgery [Ludwig 2013]. This protocol was followed in this study to evaluate whether nutritional support affect cell immunity in patients who underwent on- and off- pump 


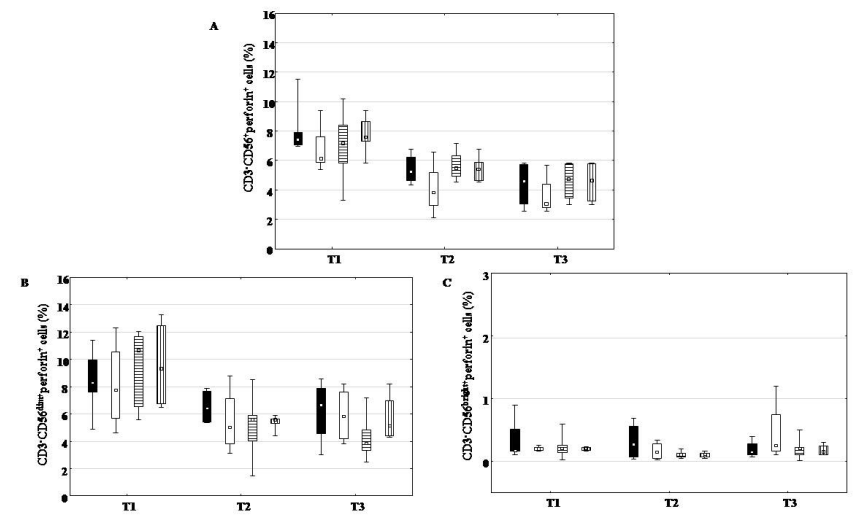

Figure 6. The frequency of perforin-positive cells within the entire CD3-CD56 cell population (A), and in the CD3-CD56 ${ }^{\text {+dim }}(B)$ or CD3CD56 $6^{\text {bright }}(\mathrm{C})$ subsets within peripheral blood lymphocytes in Group 1 (घ), Group $2(\square)$, Group $3(=)$ and Group $4(\mathrm{I})$ at times points T1 (before surgery), T2 (24 h after surgery), T3 (72 h after surgery). Data are presented as median ( $\square)$, 25th-75th percentile $(\square)$, non-outlier range $(\mathrm{I})$, and outliers $\left({ }^{\circ}\right)$. Statistical significance: ${ }^{*} P<.05$.

CABG surgery. We did not find any changes in frequency, lymphocyte subsets and their perforin expression in respect to supplementary feeding. This could be explained by the content of the preoperative drink, which lacks proteins and lipids that could affect immune response. Nutritional support with proteins accelerate postoperative recovery, as proteins maintain nitrogen balance and muscle mass [Anastasilakis 2013]. Special attention was given to types of essential fats. Fats with polyunsaturated fat acids (PUFAs), rich with omega- 3 fatty acids, have special privileges on the health of patients with ischemic heart disease, because they prevent the occurrence of major cardiovascular events and act with immunomodulation [Molfino 2014]. Omega-3 fatty acids prevent the onset of arrhythmia; and increase the cardiac autonomic modulation and the effect of antiarrhythmic therapy [Molfino 2014]. They diminish systolic blood pressure, incidence of chronic heart failure risk and heart failure-related total mortality [Molfino 2014].

From the above, some limitations of our study become evident, the most important being the nutrition formula used in this study, containing only carbohydrates but not proteins or fats; and it was given only once. The supplements containing fats and proteins are not recommended for preoperative feeding. However, the administration of such supplements could give us better information on the changes of cell response in patients undergoing CABG surgery, particularly if taking blood samples more often.

\section{Conclusion}

Preoperative oral feeding with high content carbohydrates nutrition did not have a beneficial effect of dynamic changes of frequency and perforin expression in patients undergoing CABG surgery. Further investigation with different nutritional support and regimens should be investigated to find the best preoperative strategy in order to maintain balance of neuro-immuno-endocrine axis in patients undergoing highrisk surgery such as CABG surgery.

\author{
The authors would like to thank Mrs. Ksenija Tulic for \\ technical support throughout the study.
}

\section{REFERENCES}

Abbate A, Bussani R, Sinagra G, et al. 2008. Right ventricular cardiomyocyte apoptosis in patients with acute myocardial infarction of the left ventricular wall. Am J Cardiol 102:658-62. Akasaka Y, Morimoto $\mathrm{N}$, Ishikawa $\mathrm{Y}$, et al. 2006. Myocardial apoptosis associated with the expression of proinflammatory cytokines during the course of myocardial infarction. Mod Pathol 19:588-98.

Akbas H, Erdal AC, Demiralp E, Alp M. 2002. Effects of coronary artery bypass grafting on cellular immunity with or without cardiopulmonary bypass: changes in lymphocytes subsets. Cardiovasc Surg 10:586-9.

Allavena P, Giardina G, Bianchi G, Mantovani A. 1997. IL-15 is chemotactic for natural killer cells and stimulates their adhesion to vascular endothelium. J Leukoc Biol 61:729-35.

Anastasilakis CD, Ioannidis O, Gkiomisi AI, Botsios D. 2013. Artificial nutrition and intestinal mucosal barrier functionality. Digestion 88:193-208.

Bujak M, Frangogiannis NG. 2009. The role of IL-1 in the pathogenesis of heart disease. Arch Immunol Ther Exp (Warsz) 57:165-76.

Buxton BF, Hayward PA. 2013. The art of arterial revascularization-total arterial revascularization in patients with triple vesselcoronary artery disease. Ann Cardiothorac Surg 2:543-51.

Calafiore AM, Di Mauro M, Canosa C, Di Giammarco G, Iaco AL. 2003. Contini M. Myocardial revascularization with and without cardiopulmonary bypass: advantages, disadvantages and similarities. Eur J Cardiothorac Surg 24:953-60.

Chávez-Galán L, Arenas-Del Angel MC, Zenteno E, Chávez R, Lascurain R. 2009. Cell death mechanisms induced by cytotoxic lymphocytes. Cell Mol Immunol 6:15-25.

Finegold JA, Asaria P, Francis DP. 2012. Mortality from ischaemic heart disease by country, region and age: Statistics from World Health Organisation and United Nations. Int J Cardiol 168:934-45.

Jankovicová K, Kudlová MT, Kolácková M, et al. 2008. The effect of cardiac surgery on peripheral blood lymphocyte populations. Acta Medica (Hradec Kralove) 51:25-9.

Jeremias A, Kaul S, Rosengart TK, Gruberg L, Brown DL. 2009. The impact of revascularization on mortality in patients with nonacute coronary artery disease. Am J Med 122:152-61.

Kazmierski J, Banys A, Latek J, Bourke J, Jaszewski R. 2014. Raised IL-2 and TNF- $\alpha$ concentrations are associated with postoperative delirium in patients undergoing coronary-artery bypass graft surgery. Int Psychogeriatr 26:845-55.

Laskarin G, Zaputovic L, Persic V, Ruzic A, Sotosek Tokmadzic V. 2012. Harmful immune reactions during acute myocardial infarction. Med Hypotheses 78:703-6.

Ludwig RB, Paludo J, Fernandes D, Scherer F. 2013. Lesser time of preoperative fasting and early postoperative feeding are safe? Arq Bras Cir Di 26:54-8.

Martins TB, Anderson JL, Muhlestein JB, et al. 2006. Risk factor analysis of plasma cytokines in patients with coronary artery disease by a multiplexed fluorescent immunoassay. Am J Clin Pathol 125:906-13.

Molfino A, Gioia G, Fanelli FR, Muscaritoli M. 2014. The role for dietary 
omega-3 fatty acids supplementation in older adults. Nutrients 6:4058-72.

Møller CH, Steinbrüchel DA. 2014. Off-pump versus on-pump coronary artery bypass grafting. Curr Cardiol Rep 16:455.

Orhan G, Sargin M, Senay S, et al. 2007. Systemic and myocardial inflammation in traditional and off pump cardiac surgery. Tex Heart Inst J 34:160-5.

Perera PY, Lichy JH, Waldmann TA, Perera LP. 2012. The role of interleukin-15 in inflammation and immune responses to infection: implications for its therapeutic use. Microbes Infect 14:247-61.

Persic V, Ruzic A, Miletic B, et al. 2012. Granulysin expression in lymphocytes that populate the peripheral blood and the myocardium after an acute coronary event. Scand J Immunol 78:231-42.

Pipkin ME, Lieberman J. 2007. Delivering the kiss of death: progress on understanding how perforin works. Curr Opin Immunol 19:301-8.

Solomon MD, Tirupsur A, Hytopoulos E, et al. 2013. Clinical utility of a novel coronary heart disease risk-assessment test to further classify intermediate-risk patients. Clin Cardiol 36:621-7.

Subjective Global Assessment of Nutritional Status. http://www.fpnotebook.com/Pharm/Exam/SbjctvGlblAssmntOfNtrtnlSts.htm.
Takagi H, Watanabe T, Mizuno Y, Kawai N, Umemoto T; for the ALICE (All-Literature Investigation of Cardiovascular Evidence) Group. 2014. A meta-analysis of adjusted risk estimates for survival from observational studies of complete versus incomplete revascularization in patients with multivessel disease undergoing coronary artery bypass grafting. Interact Cardiovasc Thorac Surg 18:679-82.

Takayama T, Hiro T, Hirayama A. 2010. Is angioplasty able to become the gold standard of treatment beyond bypass surgery for patients with multivessel coronary artery disease? Therapeutic strategies for 3-vessel coronary artery disease: OPCAB vs PCI (PCI-Side). Circ J 74:2744-9.

Tokmadzic VS, Tsuji Y, Bogovic T, et al. 2002. IL-18 is present at the maternal-fetal interface and enhances cytotoxic activity of decidual lymphocytes. Am J Reprod Immunol 48:191-200.

Windecker S, KolhP, Alfonso F, et al. 2014. 2014 ESC/EACTS Guidelines on myocardial revascularization. Eur Heart J 34:2541-619.

Zal B, Kaski JC, Akiyu JP, et al. 2008. Differential pathways govern CD4+CD28- $\mathrm{T}$ cell proinflammatory and effector responses in patients with coronary artery disease. J Immunol 81:5233-41.

Zuidema MY, Zhang C. 2010. Ischemia/reperfusion injury: The role of immune cells. World J Cardiol 2:325-32. 